In Crescendo. Institucional. 2015; 6(1): 22-32

\title{
FACTORES SOCIALES E INCIDENCIA DE ENTEROBIUS VERMICULARIS EN La institución educativa inicial Semillitas del Saber
}

\author{
SOCIAL FACTORS AND INCIDENCE OF ENTEROBIUS VERMICULARIS IN \\ initial edUCATIONAL institution Semillitas delSaber
}

\author{
Marisol Contreras Quiñones* \\ Juan Carlos Rodríguez Soto**
}

\begin{abstract}
RESUMEN
En n el presente estudio se tuvo como objetivos determinar los factores sociales (hábitos de higiene personal y saneamiento básico intradomiciliario) y su relación con la incidencia de Enterobius vermicularis en infantes de la institución educativa de nivel inicial Semillitas del saber, del distrito de Poroto, La Libertad. La muestra estuvo conformada por 41 infantes de la única institución educativa inicial del distrito de Poroto, Semillitas del saber. Se aplicó la prueba de Graham, una vez por niño, y se encontró que el 29,27 $\%$ de los niños estaban parasitados por Enterobius vermicularis, de los cuales el 75 \% son hombres, $25 \%$ mujeres y 58,33 \% de 4 años de edad. En la población de infantes de nivel inicial de Poroto, los factores sociales de higiene personal y saneamiento básico intradomiciliario, en su mayoría fueron buenos, por lo que el resultado indica que el parasitismo por Enterobius vermicularis resultó no estar asociado con ninguno de los factores sociales estudiados sin significancia estadística $\mathrm{p} \geq 0,05$.
\end{abstract}

Palabras claves: Enterobius vermicularis, factores sociales, infantes.

\footnotetext{
* Magíster, docente de la Universidad Católica Los Ángeles de Chimbote. maryt_27@hotmail.com

** Magíster egresado de la Universidad Católica Los Ángeles de Chimbote. juanca_113@hotmail.com
} 


\section{ABSTRACT}

We studied the social habits of personal hygiene and in-house basic sanitation, and their relation to the incidence of Enterobius vermicularis in pre-school children of Poroto district, La Libertad. The sample consisted of 41 children of pre-school Semillitas del Saber in Poroto district, It was applied the Graham test, whose results were: 29,27 \% of children were infected by Enterobius vermicularis, of which, $75 \%$ are male, $25 \%$ female and $58,33 \%$ were 4 years old. In the children population-level of Poroto, the social habits of personal hygiene and in-house basic sanitation were mostly good, so the result indicates that parasitism by Enterobius vermicularis was not associated with any of the mentioned factors, with no statistical significance $(\mathrm{p} \geq 0,05)$.

Keywords: Enterobius vermicularis, social factors, children. 


\section{INTRODUCCIÓN}

Hoy en día, los sistemas de salud, incluso en los países más desarrollados, no logran los objetivos de brindar un servicio global e integral de salud a toda la población. Aunque se han alcanzado notables progresos para mejorar la salud, luchar contra la enfermedad y prolongar la vida, las personas no están satisfechas con los sistemas de salud en todo el mundo (1).

Las enfermedades parasitarias están presentes tanto en países menos desarrollados como en los países más desarrollados, y el Perú no es ajeno a esta realidad (2). La alta incidencia de infección por parásitos intestinales y poliparasitismo afecta la salud de los individuos, pudiendo causar deficiencia en el aprendizaje y función cognitiva, principalmente en los niños, quienes son los más afectados. Las infecciones crónicas por helmintos pueden causar desnutrición crónica en el hospedero, aunque esta relación no ha sido demostrada debido a que en la desnutrición participan otros factores $(3,4,5,6)$.

Una de las enfermedades parasitarias más comunes en las personas, sobre todo a los largo de la niñez, es la oxiuriasis, producida por un parásito denominado Enterobius vermicularis (oxiuro). El Departamento de Salud del estado de Nueva York en los EE.UU. señala que la oxiuriasis es la enfermedad parasitaria más común en su población. En el Perú, las altas tasas de parasitismo intestinal reportadas por numerosos investigadores deben incentivar la creación inmediata y urgente de estrategias de control y prevención para evitar el daño que ocasionan los enteroparásitos $(3,7)$.

El distrito de Poroto no escapa a esta realidad, a lo que se agregan otros factores sociales como la carencia de agua potable las 24 horas del día, falta de educación en la prevención de enfermedades parasitarias, falta de servicios higiénicos adecuados, etc., que facilitarían la incidencia de oxiuriasis si no se hace un control y seguimiento de dicha parasitosis. El distrito de Poroto se ubica al noreste de Trujillo, a unos 50 minutos por vía terrestre, a una altitud promedio de 627 metros sobre el nivel del mar; cuenta con un clima muy cálido con temperaturas promedio de entre $25^{\circ} \mathrm{C}$ a $30^{\circ} \mathrm{C}$, dependiendo de la estación y del momento del día. En cuanto al sector Salud, solo se cuenta con un establecimiento de salud que no logra atender a la totalidad de la población de dicho distrito debido a la carencia de personal y de una adecuada infraestructura.

Este estudio se realizó en el distrito de Poroto que pertenece a la provincia de Trujillo, departamento de La Libertad, el cual contaba con tres instituciones educativas de nivel inicial que congregaban a la mayoría de infantes de entre 2 y 5 años de edad: la IEI 1647 Vitelio Solórzano Ibáñez, la IEI 1585 Semillitas del saber y la IEI 1779 La Libertad. Sin embargo, en la actualidad solo se encuentra en actividad la institución Semillitas del saber. 
No existe un estudio de investigación detallado de oxiuriasis en el distrito de Poroto; sin embargo, podemos señalar: Marcos et al. (3), Prevalencia de parasitosis intestinal en niños del Valle del Mantaro, Jauja, Perú, Cazorla y col (8), Estudio clínico-epidemiológico de enterobiasis en preescolares y escolares de Taratara, estado Falcón, Venezuela, Liñán y Jara (9), Frecuencia y aspectos epidemiológicos del parasitismo por helmintos intestinales en la población infantil de Paiján, La Libertad, Perú, Beltrán y col (10), Evaluación de los métodos de Graham y Pin Tape en el diagnóstico de E. vermicularis, Iannacone y col (11), Prevalencia de infección por parásitos intestinales en escolares de primaria de Santiago de Surco, Lima, Perú, Gutiérrez y col (12), Enfermedades del apéndice cecal, Requena y col (13), Infección por Enterobius vermicularis en niños preescolares de ciudad Bolívar, Venezuela, Guevara y col (14), Enteroparasitosis en poblaciones indígenas y mestizas de la sierra de Nayarit, México, Vidal y col (15), Evolución de la prevalencia de enteroparasitosis en la ciudad Talca, región del Maule, Chile, Soriano y col (16), Parasitosis intestinales y su relación con factores socioeconómicos y condiciones de hábitat en niños de Neuquén, Patagonia, Argentina, Pérez y col (17), Intervención educativa y parasitismo intestinal en niños de la enseñanza primaria, Medina y col (18), Prevalencia de parásitos intestinales en niños que asisten al Templo Comedor Sagrado Corazón Teresa Benedicta de la Cruz, del barrio Vallejuelos, Milano y col (19), Enteroparasitosis infantil en un área urbana del nordeste argentino, Prado y col (20), Risk factors for infection with Giardia duodenalis in pre-school children in the city of Salvador.

La oxiuriasis es una parasitosis intestinal causada por un nemátodo llamado E. vermicularis (oxiuro). Los nemátodos son parásitos con aspecto de gusanos redondos, muy pequeños, que pertenecen a la clasificación de los parásitos helmintos (gusanos). E. vermicularis (oxiuro) viene a ser un pequeño nemátodo blanquecino y delgado como un hilo (21).

La hembra mide alrededor de $1 \mathrm{~cm}$ y el macho, 0,5 cm de longitud por 0,4 y 0,6 mm de diámetro, respectivamente. La extremidad anterior termina en una expansión cuticular que le sirve al gusano como medio de fijación al intestino humano. Su extremo posterior es aguzado, en la hembra es recto y en el macho, enroscado (21). Cada hembra de oxiuro coloca alrededor de 11000 huevos, muy livianos, los cuales, luego de que se ha secado la sustancia aglutinante que los mantenía adheridos a la piel, se diseminan en la ropa interior y en la cama, el suelo y otras superficies que pueden ser incluso lejanas debido al acto de sacudir las sábanas y ropa de dormir. El hombre se infecta a través de la vía digestiva por ingestión o inhalación de los huevos del parásito. Además de la vía digestiva, se ha descrito la infección directa por vía rectal (21).

La sospecha clínica más notoria está basada en el prurito anal y nasal, acompañado de molestias nerviosas. El diagnóstico de certeza se efectúa mediante el hallazgo de los gusanos o huevos, los gusanos se identifican por su pequeño tamaño, su color blanquecino y su aspecto filiforme, lo que los diferencia de otros helmintos, proglótidos o larvas de moscas, los cuales también pueden encontrarse en las inmediaciones de la región perianal o en las deposiciones. La búsqueda de huevos se efectúa mediante la prueba de la cinta adhesiva o técnica de Graham (22). 


\section{Problema}

¿Cuáles son los factores sociales y la incidencia de Enterobius vermicularis por género y edad en infantes de la institución educativa de nivel inicial Semillitas del saber del distrito de Poroto, La Libertad?

\section{Objetivo general}

Determinar los factores sociales y la incidencia de Enterobius vermicularis en la institución educativa de nivel inicial Semillitas del saber del distrito de Poroto, La Libertad.

\section{Objetivos específicos}

Determinar la incidencia, por género y por edad, de E. vermicularis en infantes de la institución educativa de nivel inicial Semillitas del saber del distrito de Poroto, La Libertad.

Las parasitosis del aparato digestivo son muy comunes en el Perú, con gran incidencia en el departamento de Lima $(24,25)$. El 30 \% de los menores de edad, cuyas edades fluctúan entre los 2 y 10 años generalmente presentan algún tipo de endoparasitosis. Los niños en edad escolar son uno de los grupos más vulnerables frente al riesgo de adquirir enfermedades infecciosas. Una vez que un niño es infectado, la probabilidad de contagio hacia sus familiares cercanos es alta (24).

Poroto, en la actualidad, es considerada como la Ciudad de la Piña, con una alta producción, con buena calidad de este producto, lo que ha permitido que sea vista por agentes exportadores. Se está siguiendo un plan de desarrollo concertado que va desde 2007 a 2015 con la finalidad de colocar a este distrito a la vanguardia del mundo actual. Posee una población cercana a los 4000 habitantes y se muestra como un centro eje de importancia nacional. No existen investigaciones de este tipo reportadas en la población más vulnerable como son los infantes del distrito de Poroto. La detección de la incidencia de oxiuriasis permitió conocer los factores sociales de los niños afectados, que fueron derivados al centro de salud del distrito para su respectivo tratamiento, seguimiento y educación preventiva promocional específica.

\section{MATERIAL Y MÉTODO}

La presente investigación, de acuerdo con la clasificación propuesta por Hernández (26), se ajusta a un tipo cuantitativo y de diseño descriptivo correlacional de corte transversal.

La población la constituyeron los infantes de 2 a 5 años de la institución educativa de nivel inicial Semillitas del saber del distrito de Poroto, en el año 2012, con un total de 41 infantes, quienes constituyeron a la vez la muestra. Se logró muestrear a cada uno de los 
infantes, lo que permitió hacer un muestreo por conveniencia. Se utilizaron dos instrumentos, uno que consiste en la aplicación de un cuestionario a manera de encuesta acerca de los factores sociales, otro para la presencia o ausencia de oxiuros.

La técnica empleada fue la entrevista a los padres de familia. Las muestras de los infantes se analizaron con la técnica de Graham para la determinación de casos positivos o negativos.

Para aplicar el instrumento se coordinó con la directora y profesoras de la institución educativa, con el encargado del centro de salud del distrito de Cascas y con los padres de familia, cuyo consentimiento voluntario para el desarrollo de la presente investigación se buscó. Una vez terminada la recolección de datos, estos se analizaron estadísticamente con la ayuda del programa estadístico Statgraphics Plus 5.1. Se aplicó la prueba de independencia de criterios (Ji-cuadrado), con una probabilidad de error de $\leq 0,05$. Se siguió lo propuesto por Polit (27) en referencia a procedimientos éticos fundamentales.

\section{RESULTADOS}

Tabla 1. Incidencia de Enterobius vermicularis en infantes de la institución educativa del nivel inicial Semillitas del saber, de Poroto, La Libertad, 2012

\begin{tabular}{lc}
\hline Presencia de oxiuros & Institución educativa de nivel inicial Semillitas del saber \\
\hline Positivos & Cantidad \\
\hline Negativos & 12 \\
\hline Total & 29 \\
\hline
\end{tabular}

Fuente: encuesta realizada a los padres de familia de los infantes de la IEI Semillitas del saber, del distrito de Poroto en el año 2012.

Tabla 2. Distribución por género de los casos positivos de oxiuriasis en alumnos de la institución educativa del nivel inicial Semillitas del saber, de Poroto, La Libertad, 2012

\begin{tabular}{lc}
\hline & Cantidad \\
\hline Masculino & 9 \\
\hline Femenino & 3 \\
\hline Total & 12 \\
\hline
\end{tabular}

Fuente: encuesta realizada a los padres de familia de los infantes de la IEI Semillitas del saber, del distrito de Poroto en el año 2012. 
Tabla 3. Distribución por edad de los casos positivos de oxiuriasis en alumnos de la institución educativa de nivel inicial Semillitas del saber, de Poroto, La Libertad, 2012

\begin{tabular}{lc}
\hline Edad (años) & Institución educativa de nivel inicial Semillitas del saber \\
\hline 3 & Cantidad \\
\hline 4 & 5 \\
\hline 5 & 7 \\
\hline Total & 0 \\
\hline
\end{tabular}

Fuente: encuesta realizada a los padres de familia de los infantes de la IEI Semillitas del saber, del distrito de Poroto en el año 2012.

Tabla 4: Distribución de los infantes de la institución educativa del nivel inicial Semillitas del saber, según hábitos de higiene personal, del distrito de Poroto, La Libertad, 2012

\begin{tabular}{lc}
\hline Hábitos de higiene personal & Institución educativa de nivel inicial Semillitas del saber \\
\hline Muy bueno & Cantidad \\
\hline Bueno & 3 \\
\hline Regular & 3 \\
\hline Deficiente & 0 \\
\hline Muy deficiente & 0 \\
\hline Total & 41 \\
\hline
\end{tabular}

Fuente: encuesta realizada a los padres de familia de los infantes de la IEI Semillitas del saber, del distrito de Poroto en el año 2012.

Tabla 5. Distribución de los infantes de la institución educativa del nivel inicial Semillitas del saber, según saneamiento intradomiciliario, del distrito de Poroto, La Libertad, 2012 .

\begin{tabular}{lc}
\hline $\begin{array}{l}\text { Saneamiento básico } \\
\text { intradomiciliario }\end{array}$ & Institución educativa de nivel inicial Semillitas del saber \\
\hline Muy bueno & Cantidad \\
\hline Bueno & 0 \\
\hline Regular & 27 \\
\hline Deficiente & 14 \\
\hline Muy deficiente & 0 \\
\hline Total & 0 \\
\hline
\end{tabular}

Fuente: encuesta realizada a los padres de familia de los infantes de la IEI Semillitas del saber, del distrito de Poroto en el año 2012. 


\section{DISCUSIÓN}

En la tabla 1 se muestra la incidencia de E. vermicularis en infantes de la institución educativa de nivel inicial Semillitas del saber, del distrito de Poroto, La Libertad, 2012, según número y porcentaje, donde se observa que la incidencia alcanzó el 29,27 \% (12 positivos de 41 muestras). Esta incidencia coincide con los resultados de la investigación de Liñán (9), pero resulta menor que otras investigaciones realizadas en Latinoamérica: por Medina (18) en Colombia, con un $93 \%$ de parasitismo, y la de Milano (19) en la Argentina, con un $73,5 \%$ de parasitismo.

La prevalencia de oxiuriasis, verificada en nuestro medio por el método de Graham, varía de un área a otra, desde 27 \% hasta 79,5\%. Beltrán (10), en un estudio estadístico, menciona las incidencias en diversas ciudades, como Arequipa, donde se ha reportado 34,4 \% entre niños de 1,5 a 6 años de edad; en Tacna, entre escolares de 6 a 16 años se ha reportado 32,98 \%; en el distrito limeño del Rímac se halló 32,2 \%; mientras que en la ciudad de Chiclayo se informó de 27,89\%.

En la tabla 2 se muestra la distribución por género de los casos positivos de oxiuriasis en infantes de la institución educativa de nivel inicial Semillitas del saber, donde se observa que el $75 \%$ de casos positivos son del género masculino ( 9 de 12 casos positivos). La frecuencia de parasitosis intestinal en los infantes de sexo masculino fue de $75 \%$, semejantes a otros resultados nacionales encontrados (8). Esta prevalencia del sexo masculino ha sido explicada por la hiperactividad presente en los niños más que en las niñas, sumando a ello el aspecto cultural, en cuya virtud hay mayor cuidado en el aseo de los miembros del género femenino.

En la tabla 3 se muestra la distribución por edad de los casos positivos de oxiuriasis en infantes de la institución educativa de nivel inicial Semillitas del saber, del distrito de Poroto, La Libertad, 2012. En número y porcentaje, la parasitosis predominó en las edades de 3 y 4 años. Se observa que la edad de 4 años alcanzó el 58,33 \% de los casos positivos (7 de 12). La edad de 3 años alcanzó el 41,67 \% (5 de 12 positivos), y en la edad de cinco años no hubo positivo alguno. Esta situación obedece tal vez al hecho de que los padres de familia prefieran evitar la obtención de muestras de sus hijos en edades tempranas (2 a 4 años), pero aceptan estos análisis y su tratamiento en mayores edades (5 a más años).

En la tabla 4 se muestra la distribución de los infantes de la institución educativa de nivel inicial Semillitas del saber, del distrito de Poroto, La Libertad, en el año 2012, según cinco categorías del factor "hábitos de higiene personal", y se observa que la mayoría de infantes (85,36 \%) desarrolla "buenos" hábitos de higiene personal, el 7,32 \% desarrolla "muy buenos" hábitos y el 7,32 \% pertenece a la categoría "regular"; se observa, además, que las categorías "deficiente" y "muy deficiente" no poseen incidencia alguna.

En la tabla 5 se muestra la distribución de los infantes de la institución educativa de nivel inicial Semillitas del saber, del distrito de Poroto, La Libertad, en el año 2012, según cinco categorías de saneamiento básico intradomiciliario, donde se observa que la mayoría de infantes $(65,85 \%)$ del distrito de Cascas desarrolla "buenos" hábitos de higiene 
personal, y el 34,15 \% pertenece a la categoría "regular"; se observa, además, que las categorías "muy bueno", "deficiente" y "muy deficiente" no poseen incidencia alguna. La categoría "regular" constituye ya un riesgo latente para los infantes que están en ella, debiéndose tomar las acciones correspondientes que mejoren el factor "saneamiento básico intradomiciliario" del infante para colocarlo en una posición libre de riesgo.

Los factores de riesgo condicionantes en el medio ambiente (7) los agrupa en cuatro áreas según las prácticas de higiene: ingesta de agua contaminada, ingesta de alimentos contaminados, inadecuada forma de eliminación de las excretas y malos hábitos de higiene (principalmente el no lavarse las manos antes de comer, antes de preparar los alimentos, después de defecar o luego de atender al niño que ha defecado). Asimismo, el Minsa (7) manifiesta que para cada grupo etáreo existe un factor de riesgo predominante: para menores de un año se debe a malos hábitos de higiene de la madre; entre uno y cuatro se debe al saneamiento intradomiciliario y, finalmente, en los niños de cinco años o más se debe al consumo de alimentos fuera del hogar.

\section{CONCLUSIONES}

- Los factores "hábitos de higiene personal" y "saneamiento básico intradomiciliario" están asociados de manera significativa a la incidencia de E. vermicularis en los infantes de instituciones educativas de nivel inicial del distrito de Poroto, La Libertad, con una certeza del 95 \% (p < 0,05). El factor "saneamiento básico intradomiciliario" es el de mayor influencia en la incidencia de oxiuriasis en los infantes de las instituciones educativas de nivel inicial del distrito de Poroto.

- La incidencia de E. vermicularis en la institución educativa de nivel inicial del distrito de Poroto fue de 29,27\%.

- $\quad$ El mayor porcentaje (75 \%) de los casos positivos correspondió al sexo masculino.

- $\quad$ El mayor porcentaje (58,33 \%) de los casos positivos correspondió a la edad de cuatro años.

\section{REFERENCIAS BIBLIOGRÁFICAS}

(1) Organización Mundial de la Salud. Estadísticas sanitarias mundiales 2010. Suiza: OMS. 2010.

(2) Marcos, L., Maco, V., Terashima, A., Samalvides, F., Miranda, E., Gotuzzo, E. Parasitosis intestinal en poblaciones urbana y rural en Sandia, departamento de Puno, Perú. Parasitol. Latinoam. 2003; 58: 35-40. 
(3) Marcos, L., Maco, V., Terashima, A., Samalvides, F., Gotuzzo, E. Prevalencia de parasitosis intestinal en niños del Valle del Mantaro, Jauja, Perú. Rev. Med. Hered. 2002; 13 (3): 85-90.

(4) Borjas, P., Arenas, F., Angulo, Y. Enteroparasitismo en niños y su relación con la pobreza y estado nutricional. CIMEL. 2009; 14 (1): 49-54.

(5) Gutiérrez, C., Trujillo, B., Martínez, A., Pineda, A., Millán, R. Frecuencia de helmintiasis intestinal y su asociación con deficiencia de hierro y desnutrición en niños de la región occidente de México. Gac. Méd. Méx. 2007; 143 (4): 297-300.

(6) Awasthi, S., Pande, K. Prevalence of malnutrition and intestinal parasites in preschool slum children in lucknow. Indian Pediatrics. 1997; 34 (7): 599-605.

(7) Ministerio de Salud. Manual de diagnóstico y la intervención preventiva en el control de la enfermedad diarreica aguda. Lima, 2000. Perú. MINSA.

(8) Cazorla, D., Acosta, M., Zárraga, A., Morales, P. Estudio clínico epidemiológico de enterobiasis en preescolares y escolares de Taratara, estado Falcón, Venezuela. Parasitol Latinoam. 2006; 61: 43-53.

(9) Liñán, A., Jara, C. Frecuencia y aspectos epidemiológicos del parasitismo por helmintos intestinales en la población infantil de Paiján, La Libertad, Perú, entre julio de 1993 y enero de 1994.

(10) Beltrán, M., Hara, T., Tello, R. Evaluación de los métodos de Graham y Pin Tape en el diagnóstico de Enterobius vermicularis. Rev. Per. Med. Exp. Salud Pública. 2005; 22 (1): 76-78.

(11) Iannacone, J., Benites, M., Chirinos, L. Prevalencia de infección por parásitos intestinales en escolares de primaria de Santiago de Surco, Lima, Perú. Parasitol Latinoam. 2006; 61: 54-62.

(12) Gutiérrez, C., Rodríguez, A., Palenzuela, S., Beltramo, P. Enfermedades del apéndice cecal. Arch. Pediatr. Urug. 2004; 75 (1): 5-12.

(13) Requena, I., Lizardi, V., Mejía, L., Castillo, H., Devera, R. Infección por Enterobius vermicularis en niños preescolares de ciudad Bolívar, Venezuela. Rev. Biomed. 2002; 13: 231-240.

(14) Guevara, Y., Haro, I., Cabrera, M., García, G., Salazar, P. Enteroparasitosis en poblaciones indígenas y mestizas de la sierra de Nayarit, México. Parasitol Latinoam. 2003; 58: $30-34$.

(15) Vidal, S., Toloza, L., Cancino, B. Evolución de la prevalencia de enteroparasitosis en la ciudad de Talca, región del Maule, Chile. Rev. chil. infectol. 2010; 27 (4): 336-340.

(16) Soriano, S., Manacorda, A., Pierangeli, N., Navarro, M., Giayetto, A. Parasitosis intestinales y su relación con factores socioeconómicos y condiciones de hábitat en niños de Neuquén, Patagonia, Argentina. Parasitol Latinoam. 2005; 60: 154-161.

(17) Pérez, M., Sánchez, M., Cueto, G., Mayor, A., Cárdenas, N., Rodríguez, M. Intervención educativa y parasitismo intestinal en niños de la enseñanza primaria. Rev. 
Cubana Med. Gen. Integr. [Serie en internet]. 2007; 23 (2): [Alrededor de 13 pantallas]. Disponible: http://bvs.sld.cu/revistas/mgi/vol23_2_07/mgil0207.htm.

(18) Medina, L.A., García, M.G., Galván, D.A., Botero, G.J. Prevalencia de parásitos intestinales en niños que asisten al Templo Comedor Sagrado Corazón Teresa Benedicta de la Cruz, del barrio Vallejuelos, Medellín. Iatreia 2009: 22(3).

(19) Milano, A.M.F., Oscherov, E.B., Palladino, A.C., Bar, A.R. Enteroparasitosis infantil en un área urbana del nordeste argentino. Medicina (Buenos Aires) 2007 67: 238-242.

(20) Atías, A. Parasitología clínica. 4. a ed. Chile: Edit. Publicaciones Técnicas Mediterráneo; 1998.

(21) Murray, P., Rosenthal, K., Kobayashi, G., Pfaller, M. Microbiología médica. 4. a ed. España: Edit. Elsevier Sciencie; 2002.

(22) Quihui-Cota, L., Valencia, M.E., Crompton, D.W., Phillips, S., Hagan, P., Díaz-Camacho, S.P., et al. Prevalence and intensity of intestinal parasitic infections in relation to nutritional status in Mexican schoolchildren. Trans. R. Soc. Trop. Med. Hyg. 2004; 98: 653-659.

(23) Sereebutra, P., Solomons, N., Aliyu, M.H., Jolly, P.E. Sociodemographic and environmental predictors of childhood stunting in rural Guatemala. Nutr. Res. 2006; 26: 65-70.

(24) Cabrera, R. Helmintos intestinales en el Perú: análisis de la prevalencia (1981-2001). Perú/MISA/OGE- 03/039 \& Serie de Informes Técnicos de Investigación Epidemiológica. 2003. $113 \mathrm{p}$.

(25) Soriano, S.V., Manacorda, A.M., Pierangeli, N.B. Parasitosis intestinales y su relación con factores socioeconómicos y condiciones de hábitat en niños de Neuquén, Patagonia, Argentina. Parasitol Latinoam. 2005; 60: 154-61.

(26) Hernández, R., Fernández, C., Baptista, P. Metodología de la investigación. 4. a ed. México. Edit. McGraw-Hill Interamericana. 2006.

(27) Polit, D., Hungler, B. Investigación científica en ciencias de la salud. 6. a ed. México. McGraw-Hill Interamericana. 2000. 